Unfallchirurg 2015 $\cdot 118: 820$

DOI 10.1007/s00113-015-0068-x

Online publiziert: 16. September 2015

(c) Springer-Verlag Berlin Heidelberg 2015

CrossMark

\section{W. Mutschler ${ }^{1}$. C. Krettek ${ }^{2}$}

${ }^{1}$ Chirurgische Klinik und Poliklinik, Klinikum der LMU München, München, Deutschland

${ }^{2}$ Unfallchirurgische Klinik, Medizinische Hochschule Hannover (MHH), Hannover, Deutschland

\title{
Nachruf zum Tod von Prof. Dr. Dieter Nast-Kolb
}

\subsubsection{4-26.7.2015}

Wie gerne hätten wir die Verdienste von Prof. Dr. Dieter Nast-Kolb um die deutsche Unfallchirurgie z. B. anlässlich seines 65. Geburtstags gewürdigt. Stattdessen müssen wir heute an dieser Stelle seinen viel zu frühen Tod nach langer schwerer Krankheit betrauern.

Prof. Nast-Kolb war unserer Zeitschrift eng verbunden. Seit Februar 1999 war er der Herausgeber der Rubrik „Weiterbildung“, die sich durch sein Engagement als Rubrik „CME Zertifizierte Fortbildung“ $\mathrm{zu}$ einem festen und dem am meisten gelesenen Bestandteil unserer Zeitschrift entwickelt hat. Gerne war er bereit, zusätzlich ab Februar 2001 auch die Schriftleitung mit zu übernehmen, der er bis $\mathrm{Fe}$ bruar 2014 angehörte. Seine leisen und klugen Einlassungen fehlen uns seitdem.

Prof. Nast-Kolb hat sich während seines ganzen beruflichen Lebens besonders dem polytraumatisierten Patienten verpflichtet gefühlt. Diese Ausrichtung und Prägung wurde maßgeblich von seinem verehrten Lehrer und Mentor Prof. Dr. Leonhard Schweiberer beeinflusst, der 1981 auf den Lehrstuhl für Chirurgie der LMU München Innenstadt berufen wurde und dort die schon in Homburg begonnenen Arbeiten zum Polytrauma intensivierte. Prof. Nast-Kolb war bald ein besonders geschätzter Mitarbeiter und publizierte u. a. in den Heften von Der Unfallchirurg zur klinischen Wertigkeit biochemischer Faktoren beim Polytrauma (1991) und zum posttraumatischen Multiorganversagen (1996). Seine Expertise und sein Organisationsgeschick verhalfen ihm rasch zu einer führenden Rolle bei der Entwicklung und Implementierung des TraumaRegisters der DGU, wel- ches die Münchener Klinik und 5 weitere in der Polytraumaversorgung führende deutsche Kliniken 1993 begründeten. Unvergessen sind die Treffen dazu, etwa am Spitzingsee in seinen von ihm so geliebten bayerischen Bergen.

Nach der Habilitation und seiner Tätigkeit als leitender Oberarzt an der chirurgischen Universitätsklinik München Innenstadt wurde er 1998 zum Direktor der Klinik für Unfallchirurgie am Universitätsklinikum Essen berufen. Dort blieb er seinem Lebensthema treu, wovon mehr als 200 Publikationen, viele Buchbeiträge und zahlreiche Aktivitäten „seiner“ Klinik etwa bei der Entwicklung der S3-Leitlinie Polytrauma oder des Weißbuches zur Schwerstverletztenversorgung zeugen. Dazu kam u. a. sein Einsatz für die Weiterentwicklung und Neuorientierung der studentischen Lehre als Studiendekan über 2 Amtsperioden.

Prof. Nast-Kolb war als Klinikdirektor, Unfallchirurg und Arzt Vorbild durch seine ruhige und zugewandte Art, seine Verbindlichkeit, seine Zuverlässigkeit und sein Fachwissen. Sein früher Tod macht betroffen. Die Familie von Dieter NastKolb hat ihre Traueranzeige so überschrieben: „Mit dem Tod eines Menschen verliert man vieles, niemals aber die gemeinsam verbrachte Zeit." Diese gemeinsam verbrachte Zeit werden wir in ehrendem Andenken bewahren.

Unser Mitgefühl gilt seiner Familie und besonders seiner Frau, die ihm in guten und schweren Zeiten stets zur Seite stand.
Für die Herausgeber:

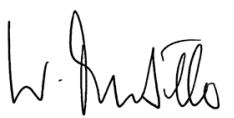

Prof. Dr. W. Mutschler<smiles>CCCC(C)C(C)C(C)C</smiles>

Prof. Dr. C. Krettek

Für den Springer-Verlag:

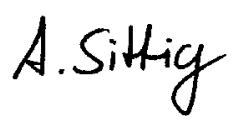

A. Sittig

\section{Korrespondenzadresse}

Prof. Dr. W. Mutschler

Chirurgische Klinik und Poliklinik Klinikum der LMU München Nußbaumstr. 20, 80336 München

Wolf.Mutschler@med.uni-muenchen.de 